LITERATURE RECEIVED

\title{
SINGLE-AGENT AND GAME-TREE SEARCH
}

\author{
T.A. Marsland \\ Technical Report TR 91-16 \\ Department of Computing Science \\ The University of Alberta \\ Edmonton, Alberta, Canada
}

We quote from the introduction:

"Problem solving by exhaustive enumeration is a common computational technique that often relies on a decision tree framework to ensure that all combinations are considered. This approach is helped by a wealth of powerful tools for supporting tree searches. A related but slightly more general model is based on a state-space approach in which, from a given state of the system and a set of actions (that is, given a description vector), the successor states are expanded until a specified goal is reached." (...)

"To illustrate these points [e.g., the limitation of storage space, the trade-off between space and time, and the use of heuristics], and to provide insights into widely applicable and generally useful techniques that can be used to improve many optimization methods, we will consider the subdomains of single agent (one person) and adversary (two-person) games. In both cases solutions can be found by traversing a decision tree that spans all the possible states in the "game". Since the order in which the decisions are made is not necessarily important, it is common for identical states to exist at different places in the decision tree. Under these circumstances such trees might be viewed as graphs. Because a tree is an intrinsically simpler structure, as well as being more regular than a graph, we will temporarily ignore such duplications, but later we will introduce methods that explicitly recognize and eliminate duplicates, and so reduce the effective size of the search space."

\section{ICCA's SPONSORS}

The production of the ICCA Journal depends on authors submitting their articles and on a secretarial staff, first guiding the submissions through the refereeing process and then taking care of the text down to the last detail. The latter is a heavy and time-consuming task.

The Editors and the Board of the ICCA gratefully recognize the SWOL Foundation (Foundation for Scientific Education in Limburg), who provided the Editor-in-Chief for 1991 again with a voluntary grant for part of the support required. Another part of the costs for Vol. 14, No. 2 up to Vol. 15, No. 1, both inclusive, has been contributed by IBM Nederland N.V.

Pursuant to our publication in last year's last issue, the Editors and Publishers of the ICCA Journal are glad to announce that two more firms have joined the comity of support:

- Mephisto, Hegener + Glaser AG of Munich, Germany, and

- Saitek Ltd. (SciSys-W Ltd.) Kwun Tong, Hong Kong.

Needless to say, ICCA and its Journal welcome them as additional sponsors with a dual emphasis. First, we are grateful to accept their material contribution; second we are happy to have been acknowledged by these sponsors as being the leading scientific Journal in the field of computer chess.

Finally, the ICCA gratefully acknowledges the four sponsorships, but stresses that not all future financial problems are solved thereby and therefore wholeheartedly welcomes new sponsors. 